\title{
Analysis of Influencing Factors for Predicting Re- intubation in Patients With Hypoxemia After Extubation: a Retrospective Study Based on the MIMIC-IV Database
}

\section{Yafen Guo}

The First People's Hospital of Changde City

Shouzhen Zhu

The First People's Hospital of Changde City

Xiangjie Duan

The First People's Hospital of Changde City

\section{Xueqing Zhang}

The First People's Hospital of Changde City

Quan Zhou

The First People's Hospital of Changde City

Jinhua Shen (D136112649@qq.com)

The First People's Hospital of Changde City

\section{Research Article}

Keywords: non-invasive ventilation, high-flow nasal cannula, reintubation rate, sequential organ failure assessment score, intensive care unit

Posted Date: March 2nd, 2022

DOI: https://doi.org/10.21203/rs.3.rs-1350742/v1

License: (c) (i) This work is licensed under a Creative Commons Attribution 4.0 International License. Read Full License 


\section{Abstract}

Purpose: The purpose of this study was to analyze the effects of high-flow nasal cannula (HFNC) and non-invasive ventilation (NIV) on the reintubation and mortality rates in adult patients with hypoxemia, and explore the risk factors for reintubation in these patients.

Patients and methods: Clinical data were extracted from the MIMIC IV database. Demographic data, vital signs, laboratory test data, and disease severity scoring systems were collected from the database. The primary and secondary outcomes were defined as the rate of reintubation and mortality at 28 days, respectively. Lasso regression was used to screen for factors influencing reintubation at 28 days in patients with hypoxemia. Receiver operating characteristic (ROC) curves were used to predict reintubation in patients with hypoxemia.

Results: In this study, 305 patients received HFNC therapy after extubation, while 234 patients received NIV treatment. Inunivariate analysis, statistically significant differences were identified in terms of respiratory support, paraplegia, sequential organ failure assessment (SOFA) score, overall anxiety severity and impairment scale (OASIS) score, pH, length of stay (LOS) in hospital, LOS in ICU, and serum potassium $(\mathrm{p}<0.05)$. In addition, we used lasso analysis and found that age, body mass index, breathing support, oxygenation index, Charlson index, $\mathrm{pH}$, serum potassium, SOFA score, and red blood cell (RBC) count can affect the occurrence of reintubation in patients with hypoxemia. The area under the ROC curves was0.77 (95\% confidence interval, $0.70-0.83$ ).

Conclusion: The 28-day reintubation and mortality rates were lower inpatients with hypoxemia treated with NIV than in those treated with HFNC. Breathing support, oxygenation index, serum potassium level, SOFA score, and RBC count were factors influencing the 28-day reintubation in patients with hypoxemia.

\section{Introduction}

About $20-30 \%$ of patients in the intensive care unit (ICU)develop respiratory failure after extubation, and nearly half of the meventually need reintubation, with the subsequent mortality rate reaching $30-40 \%$.[1, 2] Non-invasive ventilation(NIV)can generate higher gas flow and positive airway pressure, which is one of the main treatment methods for hypoxemia; however, long-term NIV can easily cause flatulence and increase the risk of aspiration and other complications.[3] Additionally, poor patient compliance because of discomfort caused by NIV often leads to treatment failure.[4] High-flow nasal cannula (HFNC) treatment is used in many patients with hypoxemia because it can quickly improve oxygenation and correct hypoxemia.[5] A number of studies have found that HFNC can improve the comfort of adult patients, increase oxygenation levels, and reduce patient dyspnea.[6, 7] However, there is no consensus on treatment results,such as intubation rate and mortality. Studies have shown that HFNC is superior to NIV in preventing reintubation and respiratory failure after extubation. [8, 9] However, some studies have shown that HFNC does notexhibit anobvious advantage in reducing reintubation rate and mortality.[10] Therefore, the purpose of this study was to investigate the effects of HFNC and NIV on the reintubation 
rate and mortality of adult patients with hypoxemia, as well as to explore the risk factors for reintubation in these patients.

\section{Material And Methods}

\section{Data Source}

The primary data for our study were derived from the MIMIC-IV database. The MIMIC-IV database is an extensive and single-center database constructed by the institutional review board of MIT and the Beth Israel Deaconess Medical Center. It contains data on admission and ICU records of more than 380,000 hospital patients admitted to the intensive care unit between 2008 and 2019. The data also includes demographic statistics, vital signs, laboratory test results, nursing staff care logs, and other information. Two of the authors obtained access to document the database after online training and were responsible for data extraction (certification ID: 6182750 and 42039823).

\section{Population selection}

Patients from 2008 to 2019 were identified in the MIMIC-IV database. The inclusion criteria for patients were as follows: (I) over 18 years of age; (II) planned extubation; (III) hypoxemia ( $100<$ arterial partial pressure of oxygen/fraction of inspiration $\left(\mathrm{PaO}_{2} / \mathrm{FiO}_{2}\right) \leq 300 \mathrm{mmHg}$ ); and (IV) having received $\mathrm{HFNC}$ or NIV therapy. The exclusion criteria were as follows: (I) prior ICU admission; (II) length of stay (LOS) in ICU less than 24 hours; (III) missing data; and (IV) having received both HFNC and NIV therapy.

\section{Study design}

The baseline characteristics of the HFNC and NIV group patients(age, sex, body mass index [BMI], and baseline disease)were collected. The documents containing data on vital signs and laboratory tests of the two groups of patients were extracted. The following vital signs were included: body temperature $(T)$, heart rate (HR), respiratory rate (RR), systolic blood pressure (SBP), diastolic blood pressure (DBP), mean blood pressure (MBP), and pulse oximetry-derived oxygen saturation $\left(\mathrm{SpO}_{2}\right)$. Laboratory test data included $\left(\mathrm{PaO}_{2} / \mathrm{FiO}_{2}\right)$; potential of hydrogen $(\mathrm{pH}) ; \mathrm{PaO}_{2} ;$ partial pressure of carbon dioxide $\left(\mathrm{PaCO}_{2}\right)$; platelet count; prothrombin time (PT); white blood cell count; red blood cell (RBC) count; and creatinine, glucose, serum potassium, and serum sodium levels. The severity scoring system included the Charlson Comorbidity Index, $\mathrm{PaO}_{2} / \mathrm{FiO}_{2}$, sequential organ failure assessment (SOFA), overall anxiety severity and impairment scale (OASIS), and simplified acute physiology score II (SAPSII). The primary outcome was 28-day reintubation. Secondary outcomes included 28-day mortality, LOS in hospital, and LOS in the ICU. In addition, our study focused on the predictors of intubation after extubation in patients with hypoxemia.

\section{Statistical analysis}

Continuous variables were expressed as means (standard deviation [SD]), and categorical covariates were expressed as numbers (percentages). The areas under the receiver operating characteristic curve (AUCs) of the predictors for the 28-day reintubation rate were calculated. To determine the risk factors for 
reintubation in patients with hypoxemia, Lasso regression was used to screen the predictors of non-zero coefficients from baseline data and test indicators. Thereafter, the screened variables were considered as independent variables and reintubation done as adependent variable to establish a logistic regression model. Receiver operating characteristic (ROC) curves constructed using bootstrap resampling (times = 500 ) were used to find the optimal cut-off values for the risk factors for reintubation. All statistical analyses were performed with Empower Stats version 2.2(http://www.empowerstats.net/,X\&Y Solutions, Inc, Boston, MA), and $p<0.05$ was considered statistically significant.

\section{Results}

\section{Patients Characteristics}

As shown in Fig. 1, the MIMIC-IV database included 19,846 adult patients with hypoxemia, of which539 patients were finally included in this study. In this study, 305 patients received HFNC therapy after extubation, while 234 patients received NIV treatment. The mean (SD) age was 67.23 (12.67) years. The mean (SD) ages were 67.03 (11.57) and67.38 (13.47) years in NIV and HFNC groups, respectively. In addition, $67.53 \%$ and $32.47 \%$ of the participants were women in NIV and HFNC group, respectively. No statistically significant differences were identified in terms of the baseline characteristics of age, sex, SAPS II, and Charlson Comorbidity Index between the two groups ( $p>0.05)$. However, at 28 days of reintubation and 28 days of mortality, HFNC had a higher risk $(p<0.05)$, as shown in Table 1. 
Table 1

Baseline characteristics of participants stratified by ventilation status

\begin{tabular}{|c|c|c|c|}
\hline Variables & $\begin{array}{l}\text { Total } \\
n=539\end{array}$ & $\begin{array}{l}\text { NIV group } \\
n=234\end{array}$ & $\begin{array}{l}\text { HFNC group } \\
n=305\end{array}$ \\
\hline Age, median mean (SD) & $67.23(12.67)$ & $67.03(11.57)$ & $67.38(13.47)$ \\
\hline \multicolumn{4}{|l|}{ Gender } \\
\hline Male, n (\%) & $364(67.53 \%)$ & $163(69.66 \%)$ & $201(65.90 \%)$ \\
\hline Female, n (\%) & $175(32.47 \%)$ & $71(30.34 \%)$ & $104(34.10 \%)$ \\
\hline BMI, mean (SD) & 32.89 (9.69) & $36.72(11.22)$ & $29.95(7.05)$ \\
\hline \multicolumn{4}{|l|}{ Baseline disease } \\
\hline Myocardial infarctionn(\%) & $143(26.53 \%)$ & $58(24.79 \%)$ & $85(27.87 \%)$ \\
\hline $\begin{array}{l}\text { Congestive heart failure, } \mathrm{n} \\
(\%)\end{array}$ & $188(34.88 \%)$ & $89(38.03 \%)$ & $99(32.46 \%)$ \\
\hline $\begin{array}{l}\text { Peripheral vascular disease, } \\
\text { n (\%) }\end{array}$ & $134(24.86 \%)$ & $36(15.38 \%)$ & $98(32.13 \%)$ \\
\hline $\begin{array}{l}\text { Cerebrovascular disease, } \mathrm{n} \\
(\%)\end{array}$ & $57(10.58 \%)$ & $19(8.12 \%)$ & $38(12.46 \%)$ \\
\hline Dementia, n (\%) & $9(1.67 \%)$ & $3(1.28 \%)$ & $6(1.97 \%)$ \\
\hline $\begin{array}{l}\text { Chronic pulmonary disease, } \\
\text { n (\%) }\end{array}$ & $205(38.03 \%)$ & $105(44.87 \%)$ & $100(32.79 \%)$ \\
\hline Rheumatic disease, n (\%) & $16(2.97 \%)$ & $7(2.99 \%)$ & $9(2.95 \%)$ \\
\hline Peptic ulcer disease, n (\%) & $9(1.67 \%)$ & $5(2.14 \%)$ & $4(1.31 \%)$ \\
\hline Liver disease, n (\%) & $54(10.02 \%)$ & $15(6.41 \%)$ & $39(12.79 \%)$ \\
\hline Diabetes, n (\%) & $165(30.61 \%)$ & $83(35.47 \%)$ & $82(26.89 \%)$ \\
\hline Paraplegia, n (\%) & $10(1.86 \%)$ & $3(1.28 \%)$ & $7(2.30 \%)$ \\
\hline Renal disease, n (\%) & $130(24.12 \%)$ & $56(23.93 \%)$ & $74(24.26 \%)$ \\
\hline Malignant cancer, n (\%) & $40(7.42 \%)$ & $12(5.13 \%)$ & $28(9.18 \%)$ \\
\hline Charlson Comorbidity Index & $6.00(4.00-8.00)$ & $6.00(4.00-7.00)$ & $6.00(4.00-8.00)$ \\
\hline $\mathrm{PaO} 2 / \mathrm{FiO} 2$, mean (SD) & 198.76 (49.99) & $211.43(50.65)$ & $189.03(47.31)$ \\
\hline
\end{tabular}

Note: * is $p<0.05$, NIV: noninvasive ventilation. HFNC: high-fow nasal cannula. BMI: body mass index. $\mathrm{PaO2} / \mathrm{FiO2}$ : arterial partial pressure of oxygen/fraction of inspiration 02. SOFA: sequential organ failure assessment. OASIS: overall anxiety severity and impairment scale. SAPSII: simplified acute physiology score II. MBP: mean blood pressure. SpO2: blood oxygen saturation. $\mathrm{pH}$ : potential of hydrogen. PaCO2: partial pressure of carbon dioxide. LOS: length of stay. ICU: intensive care unit. 


\begin{tabular}{|c|c|c|c|}
\hline Variables & $\begin{array}{l}\text { Total } \\
n=539\end{array}$ & $\begin{array}{l}\text { NIV group } \\
n=234\end{array}$ & $\begin{array}{l}\text { HFNC group } \\
n=305\end{array}$ \\
\hline SOFA score, mean (SD) & $7.64(3.20)$ & $7.05(3.00)$ & $8.09(3.28)$ \\
\hline OASIS score, mean (SD) & $36.26(8.52)$ & $35.12(8.57)$ & $37.13(8.39)$ \\
\hline SAPSII score, mean (SD) & $41.54(13.42)$ & $40.32(12.96)$ & $42.48(13.71)$ \\
\hline Heart rate, mean (SD) & $81.89(15.40)$ & $82.40(14.15)$ & $81.50(16.31)$ \\
\hline Respiratory rate, mean (SD) & $18.36(4.58)$ & $18.06(4.25)$ & $18.59(4.81)$ \\
\hline MBP, mean (SD) & $76.36(15.53)$ & 74.87 (11.77) & $77.51(17.81)$ \\
\hline Sp02,mean (SD) & $97.22(2.61)$ & $97.58(2.63)$ & $96.94(2.57)$ \\
\hline Tempreture,mean (SD) & $37.10(0.61)$ & $37.06(0.46)$ & $37.14(0.70)$ \\
\hline $\mathrm{pH}$, mean (SD) & $7.39(0.06)$ & $7.38(0.06)$ & $7.40(0.07)$ \\
\hline PaO2, median [Q1, Q3] & $95.00(80.00-122.50)$ & $\begin{array}{l}102.00(83.25- \\
134.00)\end{array}$ & $\begin{array}{l}91.00(80.00- \\
117.00)\end{array}$ \\
\hline PaCO2, mean (SD) & $42.39(8.81)$ & $44.70(10.06)$ & $40.62(7.25)$ \\
\hline $\begin{array}{l}\text { Duration before extubation, } \\
\text { median }\end{array}$ & $62.50(29.88-158.81)$ & $\begin{array}{l}51.13(22.70- \\
91.78)\end{array}$ & $\begin{array}{l}162.32(105.54- \\
227.13)\end{array}$ \\
\hline $\begin{array}{l}\text { LOS in hospital, median } \\
\text { [Q1, Q3] }\end{array}$ & $11.92(7.53-17.76)$ & $10.46(6.60-13.89)$ & $14.16(8.21-21.00)$ \\
\hline LOS in ICU, median [Q1, Q3] & $5.30(3.21-10.87)$ & $4.05(2.19-7.91)$ & $6.91(4.15-13.32)$ \\
\hline Platelets & $172.00(131.00-232.00)$ & $\begin{array}{l}173.00(135.25- \\
235.50)\end{array}$ & $\begin{array}{l}171.00(129.00- \\
231.00)\end{array}$ \\
\hline Lymphocytes & $14.00(8.30-21.75)$ & $\begin{array}{l}13.85(8.63- \\
21.78)\end{array}$ & $14.24(8.00-21.53)$ \\
\hline Hemoglobin & $\begin{array}{l}10.61(2.26) 10.40 \\
(8.90-12.00)\end{array}$ & $10.48(2.17)$ & $10.71(2.33)$ \\
\hline \multirow[t]{2}{*}{ Variables } & Total & NIV group & HFNC group \\
\hline & $n=539$ & $\mathrm{n}=234$ & $\mathrm{n}=305$ \\
\hline Hematocrit & $32.31(6.83)$ & $32.13(6.62)$ & $32.45(6.99)$ \\
\hline Prothrombin time,PT & $14.70(13.10-16.60)$ & $\begin{array}{l}14.95(13.40- \\
16.67)\end{array}$ & $\begin{array}{l}14.60(13.00- \\
16.50)\end{array}$ \\
\hline \multicolumn{4}{|c|}{$\begin{array}{l}\text { Note: * is } p<0.05 \text {, NIV: noninvasive ventilation. HFNC: high-fow nasal cannula. BMI: body mass index. } \\
\text { PaO2/FiO2: arterial partial pressure of oxygen/fraction of inspiration O2. SOFA: sequential organ } \\
\text { failure assessment. OASIS: overall anxiety severity and impairment scale. SAPSIl: simplified acute } \\
\text { physiology score II. MBP: mean blood pressure. SpO2: blood oxygen saturation. pH: potential of } \\
\text { hydrogen. PaCO2: partial pressure of carbon dioxide. LOS: length of stay. ICU: intensive care unit. }\end{array}$} \\
\hline
\end{tabular}




\begin{tabular}{|c|c|c|c|}
\hline Variables & $\begin{array}{l}\text { Total } \\
n=539\end{array}$ & $\begin{array}{l}\text { NIV group } \\
n=234\end{array}$ & $\begin{array}{l}\text { HFNC group } \\
n=305\end{array}$ \\
\hline White blood cell count & 12.00 (8.65-16.55) & $12.50(9.00-16.88)$ & $\begin{array}{l}11.80(8.50- \\
16.10)\end{array}$ \\
\hline Red blood cell count & $3.57(0.79)$ & $3.52(0.75)$ & $3.60(0.81)$ \\
\hline Creatinine & $1.00(0.80-1.40)$ & $1.00(0.80-1.30)$ & $1.00(0.80-1.40)$ \\
\hline Glucose & $127.00(106.00-155.50)$ & $\begin{array}{l}124.00(105.00- \\
156.00)\end{array}$ & $\begin{array}{l}128.00(107.00- \\
155.00)\end{array}$ \\
\hline Sodium & $138.97(3.87)$ & $139.00(3.81)$ & $138.94(3.92)$ \\
\hline Potassium & $4.37(0.71)$ & $4.43(0.75)$ & $4.32(0.68)$ \\
\hline Reintubation 28 day & $41(7.61 \%)$ & $9(3.85 \%)$ & $32(10.49 \%)$ * \\
\hline Mortality 28 days & $58(10.76 \%)$ & $18(7.69 \%)$ & $40(13.11 \%)$ * \\
\hline \multicolumn{4}{|c|}{$\begin{array}{l}\text { Note: * is } p<0.05 \text {, NIV: noninvasive ventilation. HFNC: high-fow nasal cannula. BMI: body mass index. } \\
\text { PaO2/FiO2: arterial partial pressure of oxygen/fraction of inspiration O2. SOFA: sequential organ } \\
\text { failure assessment. OASIS: overall anxiety severity and impairment scale. SAPSIl: simplified acute } \\
\text { physiology score II. MBP: mean blood pressure. SpO2: blood oxygen saturation. pH: potential of } \\
\text { hydrogen. PaCO2: partial pressure of carbon dioxide. LOS: length of stay. ICU: intensive care unit. }\end{array}$} \\
\hline
\end{tabular}

\section{Univariate analysis of reintubation in hypoxemia patients}

Table 2 shows that in univariate analysis, respiratory support with HFNC increased the reintubation risk of patients with hypoxemia [odds ratio: 2.93; 95\% confidence interval (CI):1.37-6.27; $p<0.05$ ]. Statistically significant differences were identified in terms of paraplegia, SOFA score, OASIS score, pH, LOS in hospital, LOS in ICU, and potassium level $(p<0.05)$. 
Table 2

Analysis of influencing factors of reintubation in hypoxemia patients

\begin{tabular}{|c|c|c|}
\hline \multirow[t]{2}{*}{ Variable } & \multirow[t]{2}{*}{ Statistics } & Univariate \\
\hline & & $\beta(95 \% \mathrm{Cl})$ \\
\hline \multicolumn{3}{|l|}{ Gender, n(\%) } \\
\hline Female & $175(32.47 \%)$ & 1 \\
\hline Male & $364(67.53 \%)$ & $1.18(0.58,2.36)$ \\
\hline Age,mean $\pm S$ & $67.23 \pm 12.67$ & $1.01(0.99,1.04)$ \\
\hline BMI, mean (SD) & $32.62 \pm 9.80$ & $0.96(0.93,1.00)$ \\
\hline \multicolumn{3}{|c|}{ Respiratory support,n(\%) } \\
\hline NIV & $234(43.41 \%)$ & Ref \\
\hline HFNC & $305(56.59 \%)$ & $2.93(1.37,6.27)$ * \\
\hline \multicolumn{3}{|l|}{ Baseline disease } \\
\hline \multicolumn{3}{|c|}{ Myocardial infarctionn(\%) } \\
\hline No & $396(73.47 \%)$ & Ref \\
\hline Yes & $143(26.53 \%)$ & $1.48(0.75,2.92)$ \\
\hline \multicolumn{3}{|c|}{ Congestive heart failure, $\mathrm{n}(\%)$} \\
\hline No & $351(65.12 \%)$ & Ref \\
\hline Yes & $188(34.88 \%)$ & $1.68(0.89,3.19)$ \\
\hline \multicolumn{3}{|c|}{ Peripheral vascular disease, $\mathrm{n}(\%)$} \\
\hline No & $405(75.14 \%)$ & Ref \\
\hline Yes & $134(24.86 \%)$ & $0.60(0.26,1.39)$ \\
\hline \multicolumn{3}{|c|}{ Cerebrovascular disease, n (\%) } \\
\hline No & $482(89.42 \%)$ & Ref \\
\hline Yes & $57(10.58 \%)$ & $1.84(0.78,4.38)$ \\
\hline \multicolumn{3}{|l|}{ Dementia, n (\%) } \\
\hline No & $530(98.33 \%)$ & Ref \\
\hline \multicolumn{3}{|c|}{$\begin{array}{l}\text { Note: * is } p<0.05 \text {.BMI: body mass index. NIV: noninvasive ventilation. HFNC: high-fow nasal cannula } \\
\text { PaO2/FiO2: arterial partial pressure of oxygen/fraction of inspiration O2. SOFA: sequential organ } \\
\text { failure assessment. OASIS: overall anxiety severity and impairment scale. SAPSII: simplified acute } \\
\text { physiology score II. MBP: mean blood pressure. SpO2: blood oxygen saturation. pH: potential of } \\
\text { hydrogen. PaCO2: partial pressure of carbon dioxide. LOS: length of stay. ICU: intensive care unit. }\end{array}$} \\
\hline
\end{tabular}




\begin{tabular}{|c|c|c|}
\hline \multirow[t]{2}{*}{ Variable } & \multirow[t]{2}{*}{ Statistics } & Univariate \\
\hline & & $\beta(95 \% \mathrm{Cl})$ \\
\hline Yes & $9(1.67 \%)$ & $1.53(0.19,12.55)$ \\
\hline \multirow[t]{2}{*}{ Variable } & \multirow[t]{2}{*}{ Statistics } & Univariate \\
\hline & & $\boldsymbol{\beta}(95 \% \mathrm{Cl})$ \\
\hline \multicolumn{3}{|c|}{ Chronic pulmonary disease, $\mathrm{n}(\%)$} \\
\hline No & $334(61.97 \%)$ & Ref \\
\hline Yes & $205(38.03 \%)$ & $0.74(0.37,1.46)$ \\
\hline \multicolumn{3}{|c|}{ Rheumatic disease, n (\%) } \\
\hline No & $523(97.03 \%)$ & Ref \\
\hline Yes & $16(2.97 \%)$ & $0.24(0.09,1,22)$ \\
\hline \multicolumn{3}{|c|}{ Peptic ulcer disease, n (\%) } \\
\hline No & $530(98.33 \%)$ & Ref \\
\hline Yes & $9(1.67 \%)$ & $1.53(0.19,12.55)$ \\
\hline \multicolumn{3}{|c|}{ Liver disease, n (\%) } \\
\hline No & $485(89.98 \%)$ & Ref \\
\hline Yes & $54(10.02 \%)$ & $0.97(0.33,2.83)$ \\
\hline \multicolumn{3}{|c|}{ Diabetes, n (\%) } \\
\hline No & $374(69.39 \%)$ & Ref \\
\hline Yes & $165(30.61 \%)$ & $1.50(0.78,2.89)$ \\
\hline \multicolumn{3}{|c|}{ Paraplegia, n (\%) } \\
\hline No & $529(98.14 \%)$ & Ref \\
\hline Yes & $10(1.86 \%)$ & $5.54(1.38,22.28)$ * \\
\hline \multicolumn{3}{|c|}{ Renal disease, n (\%) } \\
\hline No & $409(75.88 \%)$ & Ref \\
\hline Yes & $130(24.12 \%)$ & $1.17(0.57,2.40)$ \\
\hline
\end{tabular}

Note: * is $p<0.05 . \mathrm{BMI}$ : body mass index. NIV: noninvasive ventilation. HFNC: high-fow nasal cannula. $\mathrm{PaO2} / \mathrm{FiO} 2$ : arterial partial pressure of oxygen/fraction of inspiration 02. SOFA: sequential organ failure assessment. OASIS: overall anxiety severity and impairment scale. SAPSII: simplified acute physiology score II. MBP: mean blood pressure. SpO2: blood oxygen saturation. $\mathrm{pH}$ : potential of hydrogen. PaCO2: partial pressure of carbon dioxide. LOS: length of stay. ICU: intensive care unit. 


\begin{tabular}{|c|c|c|}
\hline \multirow[t]{2}{*}{ Variable } & \multirow[t]{2}{*}{ Statistics } & Univariate \\
\hline & & $\beta(95 \% \mathrm{Cl})$ \\
\hline \multicolumn{3}{|l|}{ Malignant cancer, n (\%) } \\
\hline No & $499(92.58 \%)$ & Ref \\
\hline Yes & $40(7.42 \%)$ & $1.84(0.68,4.98)$ \\
\hline Charlson Comorbidity Index & $5.91 \pm 2.57$ & $1.09(0.96,1.23)$ \\
\hline $\mathrm{PaO} 2 / \mathrm{FiO} 2$, mean (SD) & $198.76 \pm 49.99$ & $1.01(1.00,1.01)$ \\
\hline SOFA score, mean (SD) & $7.64 \pm 3.20$ & $1.17(1.06,1.28)$ * \\
\hline OASIS score, mean (SD) & $36.26 \pm 8.52$ & $1.06(1.02,1.10)$ * \\
\hline SAPSII score, mean (SD) & $41.54 \pm 13.42$ & $1.02(1.00,1.04)$ \\
\hline Heart rate, mean (SD) & $81.89 \pm 15.40$ & $1.00(0.98,1.02)$ \\
\hline Respiratory rate, mean (SD) & $18.34 \pm 4.60$ & $1.04(0.97,1.11)$ \\
\hline MBP, mean (SD) & $76.36 \pm 15.53$ & $1.00(0.98,1.02)$ \\
\hline Sp02,mean (SD) & $97.23 \pm 2.61$ & $0.98(0.87,1.11)$ \\
\hline Tempreture,mean (SD) & $37.10 \pm 0.61$ & $0.61(0.17,2.12)$ \\
\hline pH, mean (SD) & $7.39 \pm 0.06$ & $5.71(2.08,6.34)$ * \\
\hline PaO2, median [Q1, Q3] & $114.91 \pm 59.96$ & $0.99(0.99,1.00)$ \\
\hline $\mathrm{PaCO} 2$, mean (SD) & $42.39 \pm 8.81$ & $0.96(0.92,1.01)$ \\
\hline Duration before extubation, median & $7.08 \pm 44.99$ & $0.99(0.95,1.02)$ \\
\hline \multirow[t]{2}{*}{ Variable } & \multirow[t]{2}{*}{ Statistics } & Univariate \\
\hline & & $\boldsymbol{\beta}(95 \% \mathrm{Cl})$ \\
\hline LOS in hospital, median [Q1, Q3] & $15.17 \pm 12.70$ & $1.06(1.04,1.08)$ * \\
\hline LOS in ICU, median [Q1, Q3] & $8.18 \pm 7.89$ & $1.13(1.10,1.17)$ * \\
\hline Platelets & $191.04 \pm 90.71$ & $1.00(1.00,1.00)$ \\
\hline Lymphocytes & $15.68 \pm 11.62$ & $1.00(0.97,1.03)$ \\
\hline Hemoglobin & $10.61 \pm 2.26$ & $1.12(0.98,1.28)$ \\
\hline
\end{tabular}

Note: * is $p<0.05 . \mathrm{BMI}$ : body mass index. NIV: noninvasive ventilation. HFNC: high-fow nasal cannula. $\mathrm{PaO} 2 / \mathrm{FiO} 2$ : arterial partial pressure of oxygen/fraction of inspiration 02. SOFA: sequential organ failure assessment. OASIS: overall anxiety severity and impairment scale. SAPSII: simplified acute physiology score II. MBP: mean blood pressure. SpO2: blood oxygen saturation. $\mathrm{pH}$ : potential of hydrogen. PaCO2: partial pressure of carbon dioxide. LOS: length of stay. ICU: intensive care unit. 


\begin{tabular}{|c|c|c|}
\hline \multirow[t]{2}{*}{ Variable } & \multirow[t]{2}{*}{ Statistics } & Univariate \\
\hline & & $\beta(95 \% \mathrm{Cl})$ \\
\hline Hematocrit & $32.31 \pm 6.83$ & $1.04(0.99,1.09)$ \\
\hline Prothrombin time, PT & $16.10 \pm 8.71$ & $1.02(0.99,1.04)$ \\
\hline White blood cell count & $13.61 \pm 7.95$ & $0.96(0.91,1.02)$ \\
\hline Red blood cell count & $3.57 \pm 0.79$ & $1.45(0.98,2.15)$ \\
\hline Creatinine & $1.34 \pm 1.35$ & $0.96(0.74,1.26)$ \\
\hline Glucose & $143.49 \pm 72.93$ & $1.00(1.00,1.00)$ \\
\hline Sodium & $138.97 \pm 3.87$ & $1.02(0.94,1.11)$ \\
\hline Potassium & $4.37 \pm 0.71$ & $0.49(0.29,0.84)$ * \\
\hline \multicolumn{3}{|c|}{$\begin{array}{l}\text { Note: * is } p<0.05 \text {.BMI: body mass index. NIV: noninvasive ventilation. HFNC: high-fow nasal cannula. } \\
\text { PaO2/FiO2: arterial partial pressure of oxygen/fraction of inspiration O2. SOFA: sequential organ } \\
\text { failure assessment. OASIS: overall anxiety severity and impairment scale. SAPSIl: simplified acute } \\
\text { physiology score II. MBP: mean blood pressure. SpO2: blood oxygen saturation. pH: potential of } \\
\text { hydrogen. PaCO2: partial pressure of carbon dioxide. LOS: length of stay. ICU: intensive care unit. }\end{array}$} \\
\hline
\end{tabular}

\section{Variable selection}

Nine non-zero coefficients (age, BMI, breathing support, oxygenation index, Charlson index,pH, serum potassium level, SOFA scores, and RBC count) were screened using the LASSO logistic regression model in the training set (Fig. 2A and B).

\section{Prediction of re-intubation in patients with hypoxemia}

In addition, we test the lasso regression results with the generalized linear model, and the results showed that breathing support, oxygenation index, serum potassium level, SOFA scores, and RBC count can affect the occurrence of reintubation in patients with hypoxemia, as shown in Table 3. ROC curves were used to determine the optimal cut-off values for prediction of events during follow-up by using bootstrap resampling (times $=500)$, which showed an area under the curve of $0.77(95 \% \mathrm{Cl}: 0.70-0.83 ;$ Fig. 3$)$. A nomogram for predicting factors influencing reintubation in patients with hypoxemia are plotted in Fig. 4. A web calculator for predicting reintubation in patients with hypoxemia after extubation (based on the coefficients in Fig. 4) can be found at https://pendan2020206.shinyapps.io/DynNomapp/for use by clinicians. The calculator presents a graphical, numerical, model summary, as well as cut-offs. 
Table 3

Multivariate logistic regression analysis of reintubation in patients with hypoxemia after extubation

\begin{tabular}{|lcccccc|}
\hline & Estimate & $S E$ & $z$ value & $\exp ($ coef) & $95 \% \mathbf{C l}$ & $P$. value \\
\hline age & 0.02 & 0.02 & 1.18 & 1.02 & $(0.99 \sim 1.05)$ & 0.24 \\
\hline BMI & -0.01 & 0.02 & -0.51 & 0.98 & $(0.94 \sim 1.03)$ & 0.61 \\
\hline breathing support & 1.03 & 0.45 & 2.31 & 2.81 & $(1.17 \sim 6.76)$ & $<0.05$ \\
\hline oxygenation index & 0.01 & 0.00 & 2.83 & 1.01 & $(1.00 \sim 1.02)$ & $<0.01$ \\
\hline Charlson Comorbidity Index & 0.03 & 0.08 & 0.32 & 1.03 & $(0.88 \sim 1.20)$ & 0.75 \\
\hline pH & 3.05 & 2.72 & 1.12 & 21.13 & $(0.10 \sim 4410.00)$ & 0.26 \\
\hline potassium & -0.71 & 0.28 & -2.59 & 0.49 & $(0.28 \sim 0.84)$ & $<0.05$ \\
\hline SOFA scores & 0.15 & 0.05 & 2.89 & 1.16 & $(1.04 \sim 1.29)$ & $<0.01$ \\
\hline Red blood cell count & 0.49 & 0.22 & 2.22 & 1.63 & $(1.06 \sim 2.51)$ & $<0.05$ \\
\hline Note: BMI: body mass index. $\mathrm{pH}$ : potential of hydrogen. SOFA: sequential organ failure assessment. \\
\hline
\end{tabular}

\section{Discussion}

HFNC is comparable to NIV as a mode of respiratory support for patients with respiratory failure, and it significantly improves pulmonary oxygenation and corrects hypoxemia.[11]In this study, the 28-day mortality and reintubation rate of patients with hypoxemia after receiving high flow oxygen were significantly higher in the HFNC group than inthe NIV group $(p=0.044, p=0.004)$. Our result was similar to a meta-analysis published in 2017,[12] but Hernández[4] suggested that HFNC has obvious advantages over NIV. In our study, reintubation rates of the patients with hypoxemia in the HFNC and NIV groups were $10.49 \%$ and $3.85 \%$, respectively, which were lower than those reported in other studies, $[4,12]$ while 28-day mortality was also significantly lower than that reported in other studies.[13]

The incidence of reintubation of patients with hypoxemia is affected by many factors. A study[14]has shown that the reintubation rate of elderly patients is significantly higher than that of young patients. Suraseranivong recruited 127 intubated elderly patients and found a correlation between physiologically relevant parameters and reintubation in elderly patients.[14] Additionally, several studies have shown that age is an important risk factor for reintubation. $[15,16]$ In our study, we observed that age was an important factor in intubation in patients with hypoxemia. This may be related to the physiological state of the elderly patient. With an increase in age, the elderly have stiffer thorax, increased residual volume during respiration, weakened respiration, impaired gas exchange, and cannot adapt to changes in respiration after extubation, all of which increasethe risk for reintubation.[14, 17]

In addition, this study found that several metabolic parameters (blood urea nitrogen, sodium, and calcium levels and anion gap) were significantly associated with the rate of reintubation. However, our study 
found that the serum sodium did not affect the reintubation rate in patients with hypoxemia, whereas the serum potassium was significantly correlated with reintubation rates. Previous studies have shown that hemoglobin and albumin are influencing factors for reintubation,[18] and that patients with low hemoglobin levels are at an increased risk of reintubation. However, this phenomenon was not observed in our study. This may be related to the inclusion of elderly patients in Konishi's study which found that NT-proBNP was the only independent factor that could predict weaning failure in premature infants, but was unaffected by the serum potassium level.[19] However, we also observed this result using the lasso model. Electrolyte levels are closely related to mechanical ventilation. $\mathrm{Zhu}^{20}$ analyzed the relationship between different levels of electrolytes and the success rate of short-term weaning and found that compared with the group with normal potassium levels, the hyperkalemic group had a significantly lower success rate, However, there were no significant differences between the normal potassium group and hyperkalemia group.[20] Hypokalemia mainly affects electrically excited tissues, namely the heart and muscle. It can cause arrhythmia, aggravate heart failure, and even lead to cardiac arrest. It can cause the contraction ability of skeletal and smooth muscle to decrease, which manifests as myasthenia, muscle pain, and spasm, among others. Gastrointestinal tract and urinary tract smooth muscle dysfunction include abdominal distension, paralytic intestinal obstruction, constipation, and urinary retention. This study showed that potassium is an important influencing factor for reintubation in hypoxic patients, which may be related to a dysfunction in electrical excitation caused by hypokalemia.

According to an earlier study,[18]LOS in the ICU was significantly shorter in the weaning success group than in the reintubation group. In our study, LOS in the ICU was an independent factor influencing reintubation in patients with hypoxemia. The longer the stay in the ICU, greater risk of infection, which increased the risk of reintubation. In addition, we used lasso analysis and found that sex, age, serum potassium level, LOS in the hospital, LOS in the ICU, respiratory support, and $\mathrm{SPO}_{2}$ can affect therisk of reintubation in patients with hypoxemia. However, after adjusting for the factors of LOS in the ICU and hospital for this model, we found that age, serum potassium level, respiratory support, and $\mathrm{SPO}_{2} \mathrm{are} \mathrm{the}$ factors influencing reintubation in hypoxemia patients.

LIU[21]considers $\mathrm{HR} / \mathrm{SpO}_{2}$ as an easily accessible and a significant indicator for predicting treatment failure with HFNC. In our study, we found that $\mathrm{SPO}_{2}$ was a major factor influencing reintubation in patients with hypoxemia. $\mathrm{SpO}_{2}$ is the most commonly used clinical parameter that can be obtained by noninvasive methods and be continuously monitored as an effective index for judging oxygenation. It hasbeen widely used as an independent index for judging the prognosis of ARDS.[22] $\mathrm{SPO}_{2}$ directly reflects the respiratory status of the body. The normal range of $\mathrm{SpO}_{2}$ is $95 \%-100 \%$. After extubation, the gas exchange in patients with hypoxemiais impaired, and the level of $\mathrm{SPO}_{2}$ is low, which can predict the success of weaning to a certain extent. In previous studies, $\mathrm{SPO}_{2}$ was an important offline predictor.[23]

This study discusses HFNC treatments after extubation and hypoxemia NIV therapy in patients at risk of intubation, and found that age, serum potassium level, respiratory support, and $\mathrm{SPO}_{2}$ are factors 
influencing reintubation in hypoxemia patients, which can be applied to predict successful extubation in real life.

\section{Limitations}

There is a significant difference in quality between type 1 and type 2 respiratory failure, but only patients with hypoxemia were included in this study, and no distinction was made between the two types. In addition, previous studies have shown that patients with hypoxemia have a high probability of reintubation 24-48 hours after extubation, but this study only included the reintubation rate 28 days after extubation. Thus,further studies emphasizing these aspects are required.

\section{Conclusion}

Compared with HFNC, the 28-day reintubation rate and mortality of patients with hypoxemia treated with NIV were lower. Serum potassium, age, $\mathrm{SpO}_{2}$, and respiratory pattern were the factors influencing 28-day reintubation in patients with hypoxemia.

\section{Declarations}

\section{Acknowledgments}

We would like to thank the Massachusetts Institute of Technology and the Beth Israel Deaconess Medical Center for the MIMIC project.

\section{Author contributions}

YG, QZ and JS contributed equally to this work. YG, QZ and JS conceptualized the research aims, planned the analyses, and guided the literature review. XD and QZ extracted the data from the MIMIC-IV database. XZ and SZ participated in data analysis and interpretation. YG and JS wrote the first draft of the paper and the other authors provided comments and approved the final manuscript. All authors have unanimously agreed to the choice of the journal to which the article has been submitted; and agree to be accountable for all aspects of the work.

\section{Availability of data and materials}

The datasets analyzed during the current study are available in the MIMIC-IV repository, https://physionet.org/content/mimiciv/0.4/

\section{Consent for publication}

Not applicable.

\section{Ethical Approval}


Ethical approval was issued by the Ethics Committee of The First People's Hospital of Changde City (2021-268-01). The establishment of this database was approved by the Massachusetts Institute of Technology (Cambridge, MA) and Beth Israel Deaconess Medical Center (Boston, MA), and consent was obtained for the original data collection. Therefore, the need for informed consent were waived for this manuscript.

\section{Competing interests}

No conflict.

\section{Funding statement}

No funding.

\section{References}

1. Subira C, Hernandez G, Vazquez A, Rodriguez-Garcia R, Gonzalez-Castro A, Garcia C, et al. Effect of Pressure Support vs T-Piece Ventilation Strategies During Spontaneous Breathing Trials on Successful Extubation Among Patients Receiving Mechanical Ventilation: A Randomized Clinical Trial. JAMA 2019, 321(22):2175-2182.

2. Thille AW, Monseau G, Coudroy R, Nay MA, Gacouin A, Decavele M, et al. Non-invasive ventilation versus high-flow nasal oxygen for postextubation respiratory failure in ICU: a post-hoc analysis of a randomized clinical trial. Crit Care 2021, 25(1):221.

3. Xuan L, Ma J, Tao J, Zhu L, Lin S, Chen S, et al. Comparative study of high flow nasal catheter device and noninvasive positive pressure ventilation for sequential treatment in sepsis patients after weaning from mechanical ventilation in intensive care unit. Ann Palliat Med 2021, 10(6):6270-6278.

4. Hernandez G, Vaquero C, Colinas L, Cuena R, Gonzalez P, Canabal A, et al. Effect of Postextubation High-Flow Nasal Cannula vs Noninvasive Ventilation on Reintubation and Postextubation Respiratory Failure in High-Risk Patients: A Randomized Clinical Trial. JAMA 2016, 316(15):15651574.

5. Porhomayon J, El-Solh AA, Pourafkari L, Jaoude P, Nader ND. Applications of Nasal High-Flow Oxygen Therapy in Critically ill Adult Patients. Lung 2016, 194(5):705-714.

6. Chikata Y, Onodera M, Oto J, Nishimura M. FIO2 in an Adult Model Simulating High-Flow Nasal Cannula Therapy. Respir Care 2017, 62(2):193-198.

7. Kim ES, Lee H, Kim SJ, Park J, Lee YJ, Park JS, et al. Effectiveness of high-flow nasal cannula oxygen therapy for acute respiratory failure with hypercapnia. J Thorac Dis 2018, 10(2):882-888.

8. Thille AW, Muller G, Gacouin A, Coudroy R, Decavele M, Sonneville R, et al. Effect of Postextubation High-Flow Nasal Oxygen With Noninvasive Ventilation vs High-Flow Nasal Oxygen Alone on Reintubation Among Patients at High Risk of Extubation Failure: A Randomized Clinical Trial. JAMA 2019, 322(15):1465-1475. 
9. Ni YN, Luo J, Yu H, Liu D, Liang BM, Liang ZA. The effect of high-flow nasal cannula in reducing the mortality and the rate of endotracheal intubation when used before mechanical ventilation compared with conventional oxygen therapy and noninvasive positive pressure ventilation. A systematic review and meta-analysis. Am J Emerg Med 2018, 36(2):226-233.

10. Monro-Somerville T, Sim M, Ruddy J, Vilas M, Gillies MA. The Effect of High-Flow Nasal Cannula Oxygen Therapy on Mortality and Intubation Rate in Acute Respiratory Failure: A Systematic Review and Meta-Analysis. Crit Care Med 2017, 45(4):e449-e456.

11. Frat JP, Brugiere B, Ragot S, Chatellier D, Veinstein A, Goudet V, et al. Sequential application of oxygen therapy via high-flow nasal cannula and noninvasive ventilation in acute respiratory failure: an observational pilot study. Respir Care 2015, 60(2):170-178.

12. Zhao H, Wang H, Sun F, Lyu S, An Y. High-flow nasal cannula oxygen therapy is superior to conventional oxygen therapy but not to noninvasive mechanical ventilation on intubation rate: a systematic review and meta-analysis. Crit Care 2017, 21(1):184.

13. Stephan F, Barrucand B, Petit P, Rezaiguia-Delclaux S, Medard A, Delannoy B, et al. High-Flow Nasal Oxygen vs Noninvasive Positive Airway Pressure in Hypoxemic Patients After Cardiothoracic Surgery: A Randomized Clinical Trial. JAMA 2015, 313(23):2331-2339.

14. Suraseranivong $R$, Krairit $O$, Theerawit $P$, Sutherasan $Y$. Association between age-related factors and extubation failure in elderly patients. PLoS One 2018, 13(11):e0207628.

15. El Solh AA, Bhat A, Gunen H, Berbary E. Extubation failure in the elderly. Respir Med 2004, 98(7):661668.

16. Frutos-Vivar F, Ferguson ND, Esteban A, Epstein SK, Arabi Y, Apezteguia C, et al. Risk factors for extubation failure in patients following a successful spontaneous breathing trial. Chest 2006, 130(6):1664-1671.

17. Sharma G, Goodwin J. Effect of aging on respiratory system physiology and immunology. Clin Interv Aging 2006, 1(3):253-260.

18. Konishi Y, Nakata Y, Nemoto A, Ushijima M, Matsuura M. The preoperative risk factors of postoperative self-extubation in elderly patients. Int J Risk Saf Med 2019, 30(1):9-18.

19. Zhang Q, Shi ZY, Luo CH, Wang L, Zhang SS, Cheng XR, et al. Application of NT-proBNP in ventilator weaning for preterm infants with RDS. Pediatr Pulmonol 2014, 49(8):757-763.

20. Shang Z. The Evaluation of Serum Electrolyte Levels during Shortterm Weaning of Mechanical Ventilation in Patients with Chronic Obstructive Pulmonary Disease. Journal of Taizhou Polytechnic College 2019, 19(06):54-56.

21. Liu T, Zhao Q, Du B. Effects of high-flow oxygen therapy on patients with hypoxemia after extubation and predictors of reintubation: a retrospective study based on the MIMIC-IV database. BMC Pulm Med 2021, 21(1):160.

22. Sendagire C, Lipnick MS, Kizito S, Kruisselbrink R, Obua D, Ejoku J, et al. Feasibility of the modified sequential organ function assessment score in a resource-constrained setting: a prospective observational study. BMC Anesthesiol 2017, 17(1):12. 
23. Bilan N, Dastranji A, Ghalehgolab Behbahani A. Comparison of the spo2/fio2 ratio and the pao2/fio2 ratio in patients with acute lung injury or acute respiratory distress syndrome. $\mathrm{J}$ Cardiovasc Thorac Res 2015, 7(1):28-31.

\section{Figures}

382,278 patients screened in the MIMIC-IV database of which 19846 underwent planned extubation

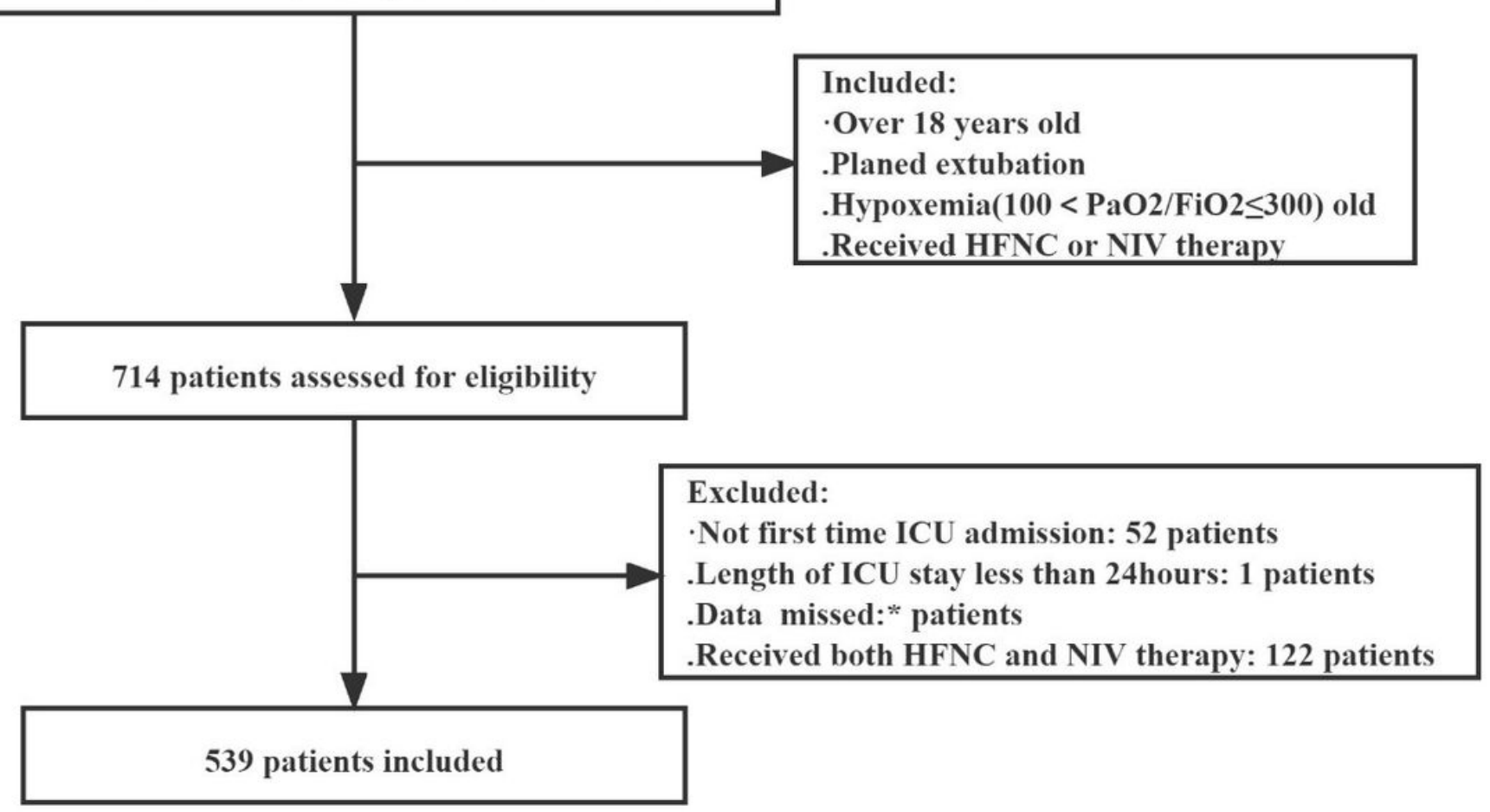

\section{Figure 1}

Flow chart of the study 

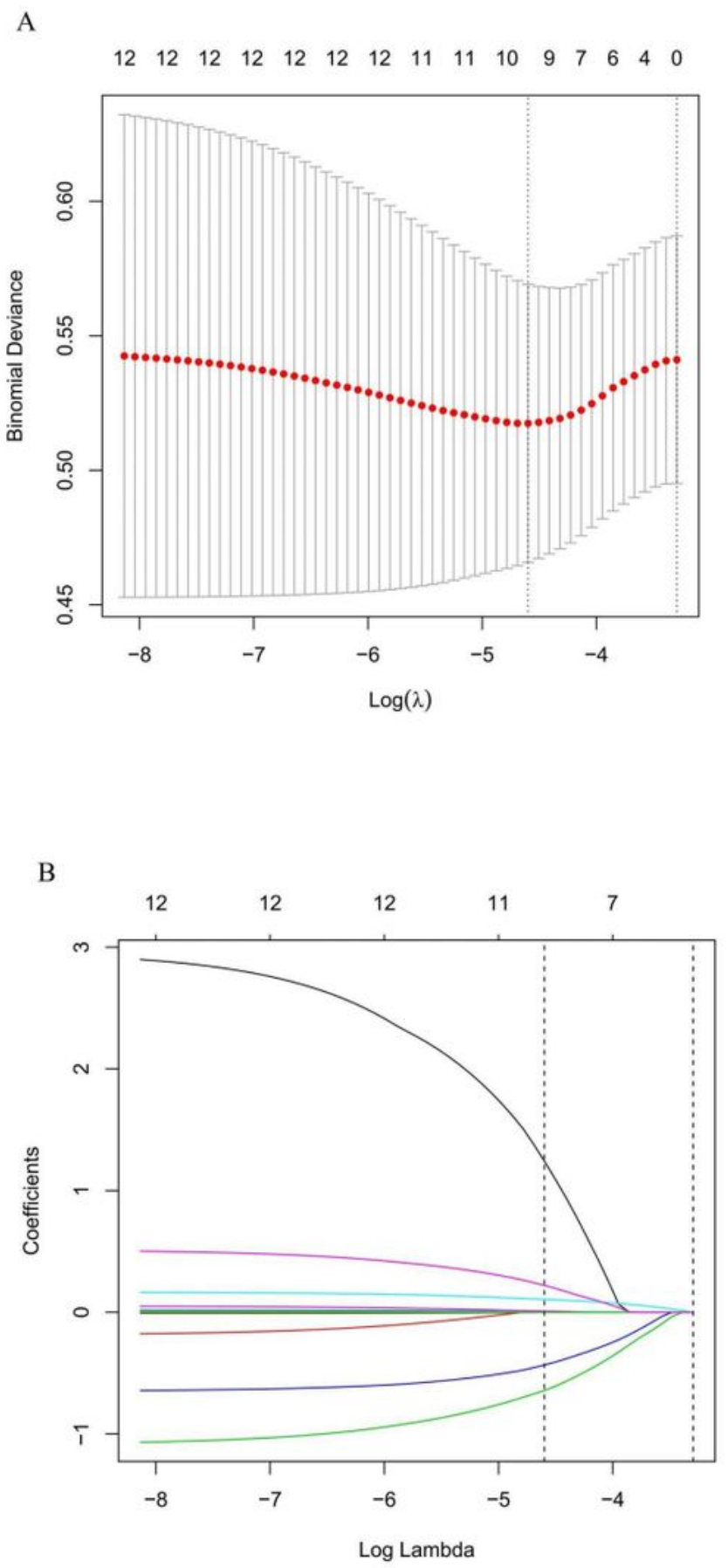

\section{Figure 2}

Texture feature selection using the least absolute shrinkage and selection operator (LASSO) binary logistic regression model A, Tuning parameter $(\lambda)$ selection in the LASSO model used 10 -fold crossvalidation via. The area under the receiver operating characteristic (AUC) curve was plotted versus log $(\lambda)$ minimum criteria. The lower $x$-ax is indicates the $\log (\lambda)$ The $y$-axis indicates binomial deviances. Dotted vertical lines were drawn at the optimal values by using the minimum criteria and the 1 standard error of 
the minimum criteria (the 1-SE criteria). A $(\lambda)$ value of 0.0101 , with $\log (\lambda)-4.5986$ was chosen (1-SE criteria) according to 10 -fold cross-validation. (B) LASSO coefficient profiles of the 150 texture features. A coefficient profile plot was produced against the $\log (\lambda)$ sequence. Vertical line was drawn at the value selected using10-fold cross-validation in $A$, where optimal $(\lambda)$ resulted in 10 nonzero coefficients.

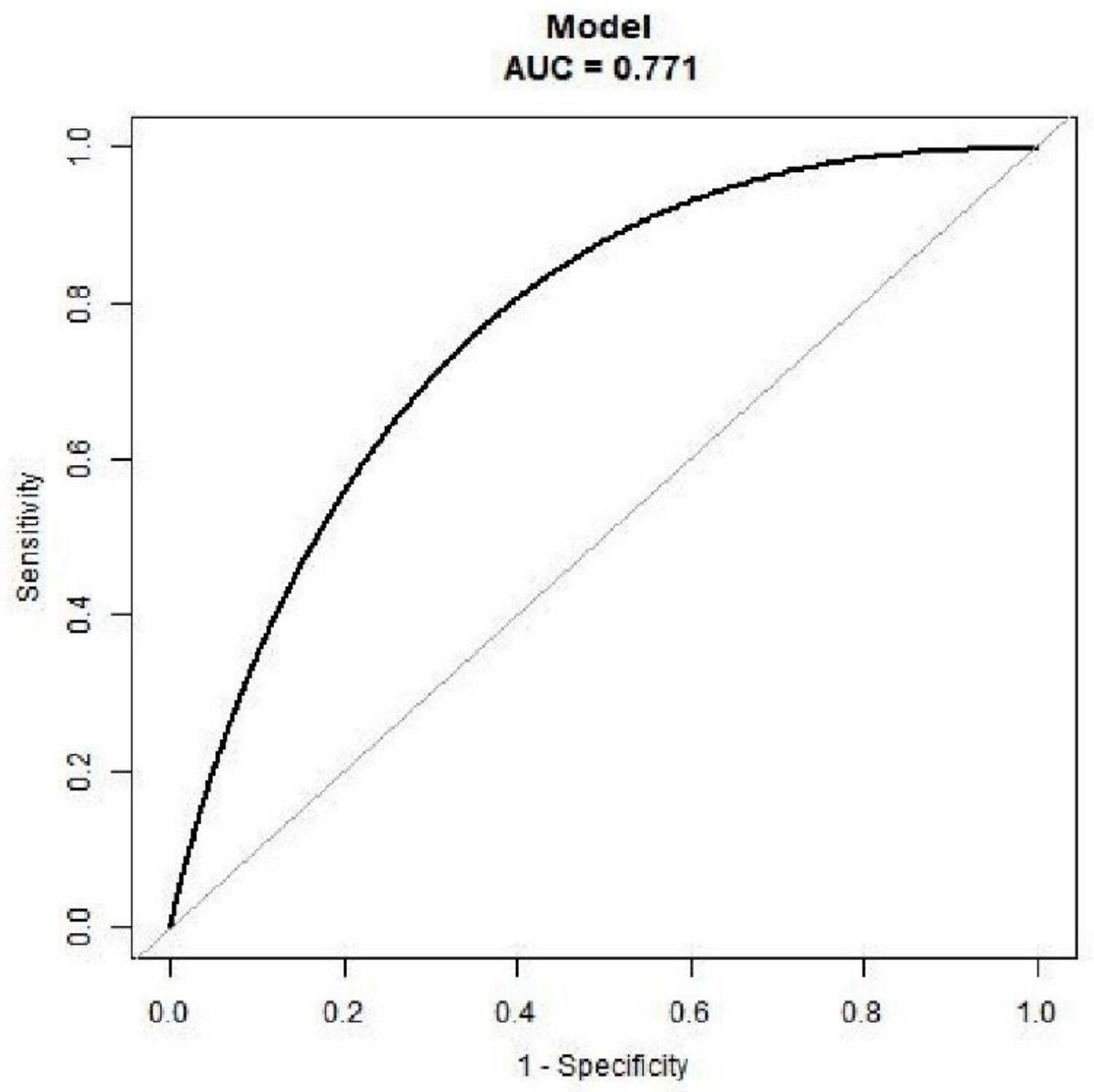

Figure 3

ROC curves for the prediction of the risk factors for reintubation in patients with hypoxemia. Using Bootstrap resampling (times $=500$ ) for the risk factors for reintubation. AUC confidence interval and 
significance tests using Bootstrap resampling. The best cut point was based on maximizing the sum of sensitivity and specificity. The analysis reveals that the percentage of the risk factors for reintubation (AUC:077, 95\%C1 0.71-0.84: sensitivity:73.2\%; specificity:72.5\%). ROC, receiver-operating characteristic: AUC, area under the curve; $\mathrm{Cl}$, confidence interval.

Points

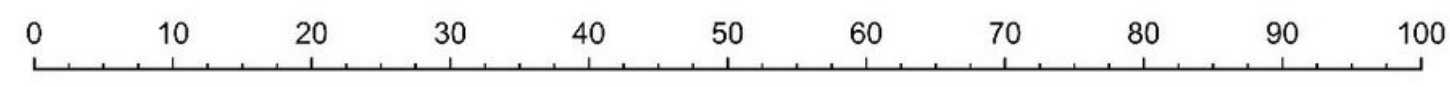

$\begin{array}{lll} & 1 \\ & & 1\end{array}$

Potassium

\begin{tabular}{lllllllllll}
\hline 8 & 7.5 & 7 & 1 & 1 & 1 & 1 & 1 & 1 & 1 & 1 \\
\hline & 1 & 6.5 & 6 & 5.5 & 5 & 4.5 & 4 & 3.5 & 3 & 2.5
\end{tabular}

SOFA scores

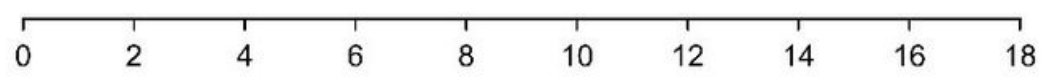

RBC.count

$\begin{array}{llllllllll}1.5 & 2 & 2.5 & 3 & 3.5 & 4 & 4.5 & 5 & 5.5 & 6\end{array}$

oxygenation index

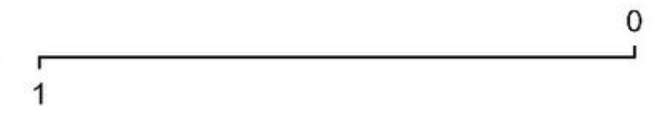

Total Points

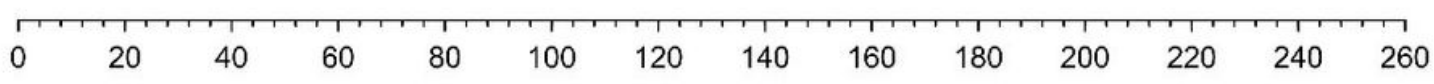

Linear Predictor

\begin{tabular}{llllllllllll}
\hline & -6.5 & -5.5 & -4.5 & -3.5 & -2.5 & -1.5 & -0.5 & 0 & 0.5
\end{tabular}

predictive probability

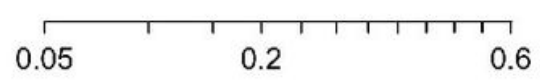

\section{Figure 4}

A nomogram for predicting factors influencing reintubation in patients with hypoxemia 